\title{
Highlighting the Deficiencies in Some Existing Optimal PMU Placement Techniques
}

\author{
Emmanuel U. Oleka ${ }^{1, *}$, Evelyn R. Sowells ${ }^{2}$, Gary L. Lebby ${ }^{1}$ \\ ${ }^{1}$ Department of Electrical and Computer Engineering, North Carolina A\&T State University, Greensboro NC 27411 \\ ${ }^{2}$ Department of Computer Systems Technology, North Carolina A\&T State University, Greensboro NC 27411 \\ *Corresponding author: eoleka@aggies.ncat.edu
}

\begin{abstract}
Synchrophasor technology is receiving a global acceptance for electric power grid Wide Area Measurement System, (WAMS), an important function in a smart power transmission grid. A critical challenge in the synchrophasor technology deployment is the optimal choice of Phasor Measurement Unit (PMU) locations on the power grid. Researchers have proposed several techniques and algorithms in this respect. This work evaluated some of the major techniques and established that the available techniques and the factors they considered are not sufficient for a real-life optimal PMU placement (OPP). It also pointed to a method that could be applied to achieve a practical and robust solution for effective PMU placement for synchrophasor applications in a real-life electric power grid. It, therefore, calls for a shift in paradigm in the studies for the optimal PMU placement locations.
\end{abstract}

Keywords: optimal PMU placement, placement considerations, synchrophasor system deployment, synchrophasor applications

Cite This Article: Emmanuel U. Oleka, Evelyn R. Sowells, and Gary L. Lebby, "Highlighting the Deficiencies in Some Existing Optimal PMU Placement Techniques." American Journal of Electrical and Electronic Engineering, vol. 5, no. 4 (2017): 120-125. doi: 10.12691/ajeee-5-4-1.

\section{Introduction}

A constant real-time monitoring of the power grid operating conditions is a requirement for the secure operation of a power system. This real-time monitoring of the power grid can be achieved with synchrophasor technology, which uses the PMUs to measure electrical waves at the different locations across the power grid and synchronizes them using a common GPS Satellite time signal. Foundational works in the development and utilization of PMU in electric power grids are found in $[1,2]$. The major challenging issues confronting the synchrophasor deployment in electric power grids were explored in [3], and they include data quality, cyber security, data transmission/channel characteristics, synchrophasor applications development, technology adaptation, PMU deployment cost, and manpower training.

Optimal PMU placement has been identified as an effective way of reducing the cost of PMU installation, ensuring efficient utilization of available transmission channel bandwidth, and enhancing the performances of the synchrophasor applications deployed into the grid. For the above reasons, researchers have devoted a lot of research energy into solving the problem of optimal PMU placement, and have developed numerous solutions to the OPP, some of which are reviewed in [4,5].

It is important to note that Optimal PMU Placement study in a power grid is supposed to be a major task that is carried out once in preparation for PMU installation on a power grid not a task for mere exercise of mathematical prowess. Hence, the OPP solution should be all encompassing, considering the existing state and possible future state of the power grid, and the characteristics inherent with the grid. The goal of optimal placement is to identify the optimal locations that maximize the benefit across multiple applications as well as offers the least-cost solution by providing for the existing and planned infrastructure upgrades across the power company's footprint and its neighboring systems [6].

\section{OPP Methods}

The rules usually considered in minimizing the number of PMUs are spelled out in [7] as follows:

- A PMU placed at a particular bus can measure the voltage phasor of that bus and the current phasors of all the branches radiating from the bus.

- If the voltage and current phasors at one end of a branch are known, the voltage phasor at the other end can be calculated using the line impedance.

- If voltage phasors at both ends of a branch are known, the branch current can be calculated using the line impedance.

- Each bus in the network must be observed at least once by PMUs.

Several solution methodologies have been proposed for the OPP problem, some of which are heuristic methods $[8,9]$, while others utilize mathematical algorithms[10-15]. Some of the solutions went further to consider one grid characteristic or another, such as the presence of contingencies, and other occurrences which are capable of 
altering the network topology and changing the optimal solution or influencing the performance of the synchrophasor system deployed.

Integer Linear Programming (ILP) approach, which was first proposed in [16], is the most common method used so far in solving the problem of OPP. The popularity of the ILP in OPP studies stems from the fact that it is simple to implement, and it gives room for modeling of some characteristics and conditions inherent in the power grids.

The optimal placement of PMUs is formulated as follows:

$$
\begin{gathered}
\text { Minimize } \sum_{i=1}^{N} c_{i} x \\
\text { s.t } A_{P M U} X \geq b_{P M U} \\
X=\left[x_{1}, x_{2}, x_{3}, \ldots x_{N}\right]^{T} \\
x_{i} \in\{0,1\}
\end{gathered}
$$

Where $c_{i}$ is the cost of PMU installation at bus $i, \mathrm{x}_{\mathrm{i}}$ is the PMU placement variable, and $A_{P M U}$ is an N-by-N matrix defined for and N-bus power grid with elements defined as

$$
a_{i, j}=\left\{\begin{array}{cc}
1 & \text { if } i=j \\
1 & \text { if } i \text { and } j \text { are connected } . \\
0 & \text { otherwise }
\end{array}\right.
$$

And $b_{\text {PMU }}$ is an N-element column vector defined as

$$
b_{P M U}=\left[\begin{array}{l}
1 \\
\cdot \\
\cdot \\
\cdot \\
1
\end{array}\right]_{\mathrm{NX} 1}
$$

\section{Placement Considerations}

Some of the necessary considerations in searching for the optimal locations of PMUs are vividly described in work in [6]. These considerations are categorized into applications, infrastructural, and operational considerations as shown in Figure 1. An OPP study should first analyze the power grid and find out which of the placement considerations apply to the grid and the intended synchrophasor applications.

\section{Deficiencies in the Existing Models}

Many of the current optimal placement models yielded solutions, which will cease to be optimal when the power grid is subjected to its practical operating conditions. Such a solution is not desirable in a predominantly data-driven electricity grid because a compromise in data quality or data availability can be of a tremendously adverse effect. The various yardsticks with which some of the models are considered insufficient are:

\section{Factors considered}

Most of the OPP formulations focused on determining the number of PMUs required for full coverage of the grid. In those formulations, the cost of PMU placement and full grid coverage were the only constraints considered. Some later models considered the fact that certain conditions and occurrences could render an observable system unobservable. They went on to consider one factor or another including contingencies, controlled islanding, etc. Figure 2 demonstrates some of the factors considered and how they are considered in some of the present OPP solution models. A close observation of the power grid still reveals that including one factor or another in the formulation is not sufficient because a regular power grid could be affected simultaneously by a combination of these factors at varying degrees. A good model should consider all these factors in their various degrees of effects they have on the synchrophasor system deployment.

Variations and distributions of characteristics

Each power grid is unique in structure and operational characteristics. For instance, some grids may have predominantly radial structures while others have loop structures. Also, some buses in the grid may be located in areas where they are susceptible to climate-induced contingencies while others are located where they play vital roles in renewable resources integrations. Some buses in a power grid may never be a candidate for locating a PMU, either due to their locations or their natures. These characteristics affect the behaviors of the grids and dictate the numbers and optimal locations of PMUs required for the synchrophasor applications on the system. An optimal placement study for a power grid can only be effective if it considers all the essential factors that affect the performances of synchrophasor applications, and the way the factors are distributed on the power grid. Figure 3 [17] shows a transmission network with some of the various occurrences that can affect the behavior of the network. These occurrences are also the factors that should be considered in PMU placement. Figure 4 gives an illustration of a distribution of power grid characteristics. For a network that is characterized as depicted in Figure 4, an OPP formulation should be able to model the three broad considerations plus the cost of placement as applies to the individual buses in the grid to yield a truly optimal solution.

Non-uniformity in the cost of placement at the buses

Most of the placement models assumed a uniform PMU installation cost for all of the buses in the system. The report in [18] identified the different factors that contribute to the effective cost of PMU placement per bus on the power grid. Analysis of those factors shows that the cost of PMU placement varies from one bus to another. The differences in placement cost per bus are often so significant that neglecting them poses significant effects on the accuracy of the resulting solutions. It follows; therefore, that optimal placement should not assume a uniform placement cost per bus but should consider the placement costs as they occur. The Per Unit (P.U.) placement cost for each of the buses in the grid can be established by taking the lowest placement cost estimated in a bus in the grid as the base cost and use it to divide the placement costs estimated for all the buses in the grid. 


\begin{tabular}{|c|c|c|}
\hline Application Considerations & Infrastructural Considerations & Operational Considerations \\
\hline State Estimation & PMU-Ready Equipment & Full Observability \\
\hline Critical Paths & Aggregate Sites & Measurement Reliability \\
\hline Local and Inter-Area Oscillation & NERC PRC 002 Sites & Security and Sensitivity \\
\hline Major Generation and Load & On Critical Cyber Asset (CCAS) List & Outage of a Line \\
\hline Renewable Integration & Channel Limits & Outage of a PMU \\
\hline Adaptive Protection & & Bad Data Consideration \\
\hline Islanding, Separation and Restoration & & Zero Injection Buses \\
\hline \multirow[t]{3}{*}{ FACTS Controllers } & & Bad Data Detection \\
\hline & & Accuracy of State Estimation \\
\hline & & Conventional Measurements \\
\hline
\end{tabular}

Figure 1. Some necessary OPP solution considerations

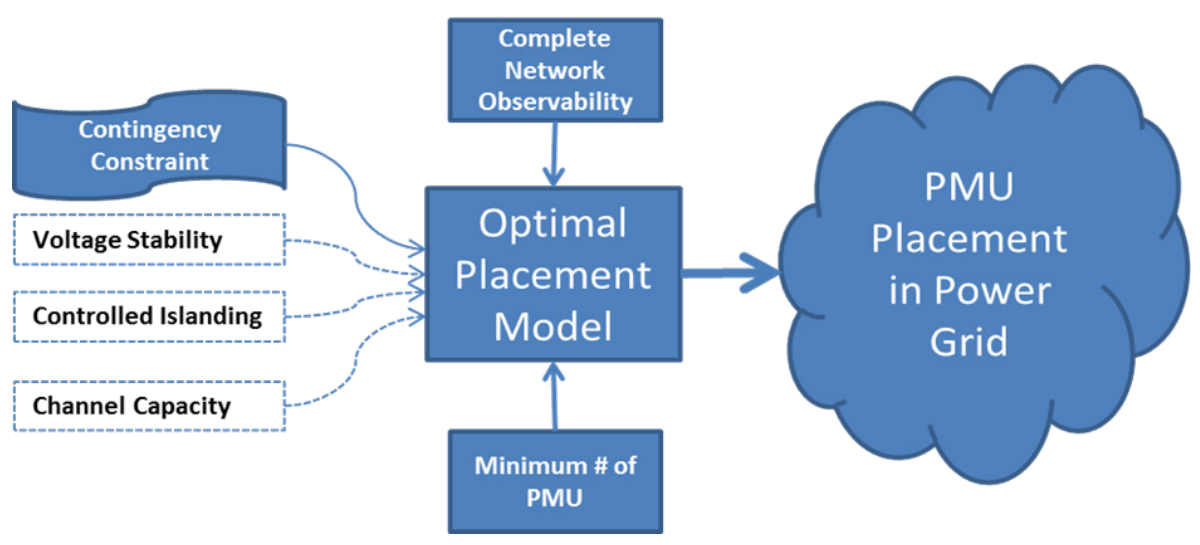

Figure 2. A demonstration of factors considered in available OPP models

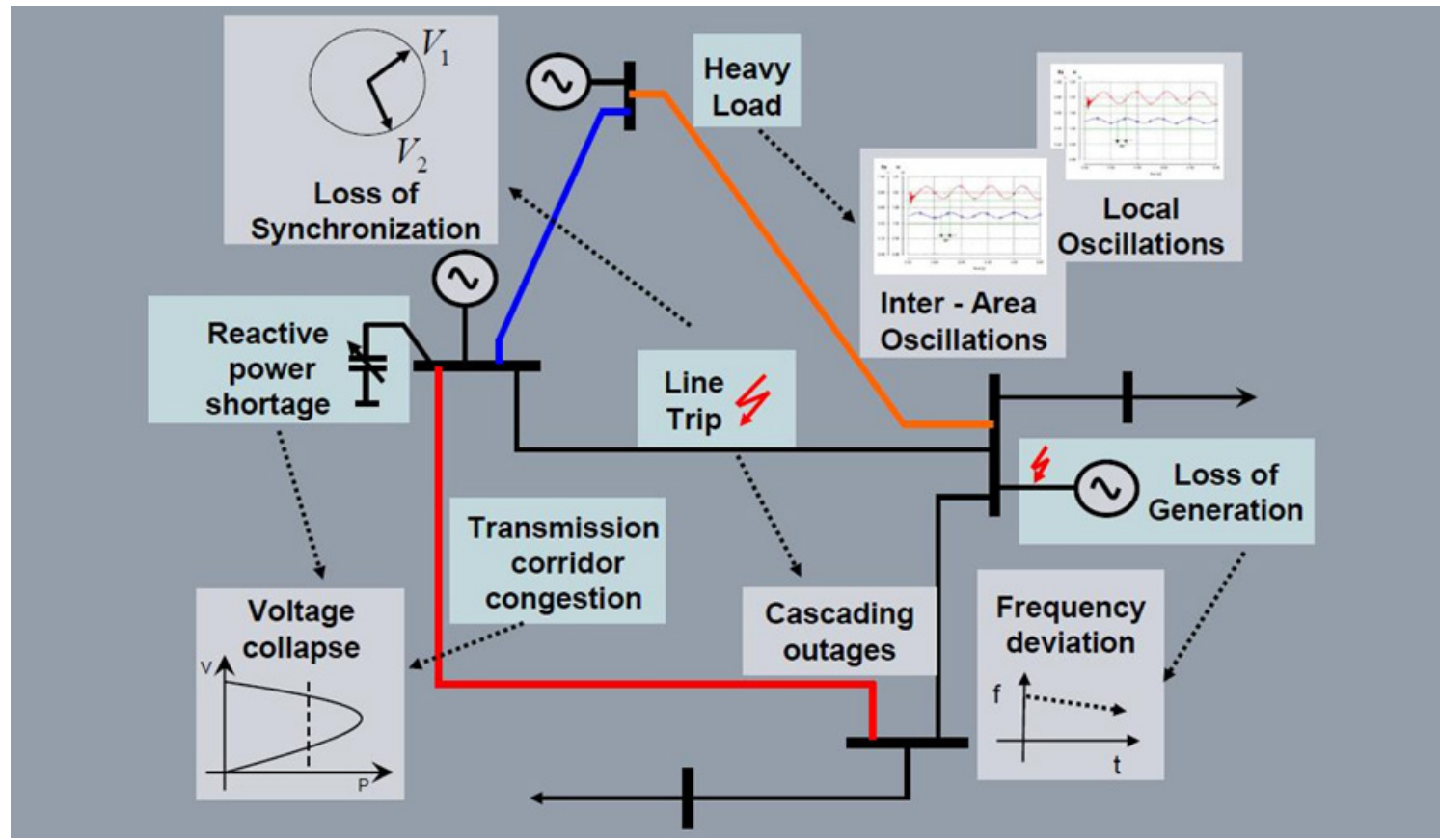

Figure 3. An example of the distribution of a network's monitoring needs [17] 


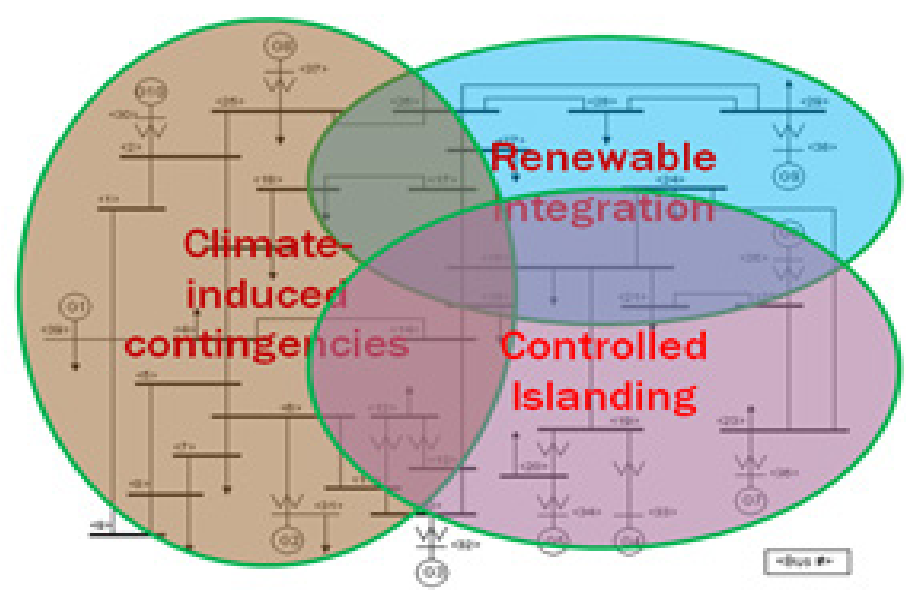

Figure 4. Example of distribution of placement factors

\section{Demonstration with IEEE Test Systems}

The claim for insufficiencies of some of the existing OPP solution models is demonstrated using IEEE Test Systems (IEEE 14-Bus System and IEEE 30-Bus System). The IEEE 14-Bus system is shown in Figure 5. The popular solutions achieved for these test systems are given in Table 1. The test systems were subjected to different contingency conditions to observe what happened to the system observability. The Test Systems were also subjected to a condition of non-uniformity in the PMU placement cost per bus. The results are shown in Table 2 to Table 6. Table 7 shows that modeling one consideration or another into the OPP model does not guarantee an adequate optimal solution because the sets of solutions achieved with the different considerations differ from one another and do not satisfy the constraints of one another.

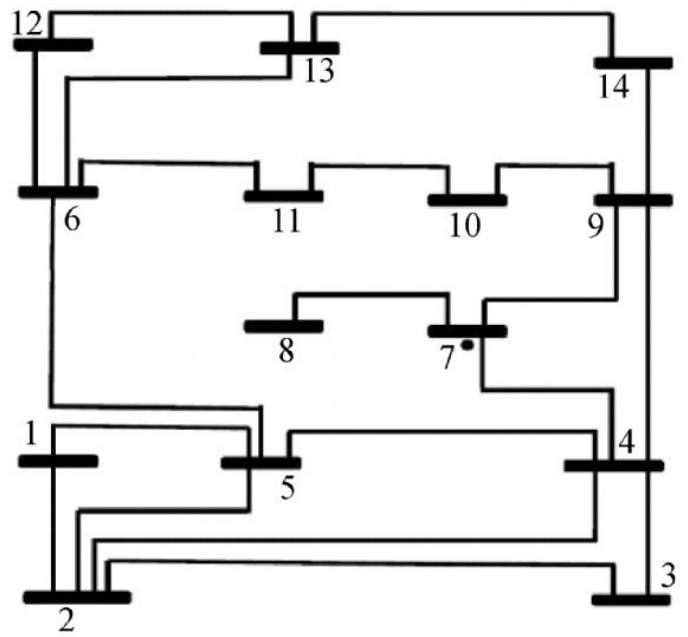

Figure 5. IEEE-14 Bus System

Table 1. Common OPP solutions for IEEE test systems

\begin{tabular}{ccc}
\hline System & General Solution & Few References \\
\hline IEEE 14-Bus & $2,7,10,12$ or $2,6,7,9$ & Ref[8], Ref[9], Ref[10] Ref[11], Ref[12], Ref[13], Ref[14], Ref[15] \\
IEEE 30-Bus & $1,2,6,9,10,12,15,19,25,27$ & Ref[14], Ref[15] \\
\hline
\end{tabular}

Table 2. Effects of contingency locations on OPP solutions for ieee-14 bus system

\begin{tabular}{|c|c|c|c|c|c|}
\hline Grid State & $\begin{array}{l}\text { Placement } \\
\text { Solution }\end{array}$ & $\begin{array}{c}\text { Unobserved } \\
\text { Bus }\end{array}$ & $\begin{array}{l}\text { Number } \\
\text { of PMUs }\end{array}$ & Solution & Comment \\
\hline Normal & $2,7,10,13$ & None & 4 & & All buses were observed \\
\hline Contingency (Line 6-13) & & 6 & 4 & $2,7,11,13$ & Bus 6 became unobserved, and solution has to change \\
\hline Contingency (Line 2-5) & & 5 & 4 & $2,6,7,9$ & Bus 5 became unobserved, and solution has to change \\
\hline Contingency (Line 9-10) & & None & 4 & $2,7,11,13$ & $\begin{array}{l}\text { All buses were still observed but a new optimal } \\
\text { solution still emerged }\end{array}$ \\
\hline Contingency (Line 1-2) & & 1 & 5 & $4,5,8,11,13$ & $\begin{array}{l}\text { Bus } 1 \text { became unobserved, and the solution has to } \\
\text { change to } 5 \text { buses }\end{array}$ \\
\hline Contingency (Line 2-3) & & 3 & 5 & $4,5,8,11,13$ & $\begin{array}{l}\text { Bus } 3 \text { became unobserved, and the solution has to } \\
\text { change to } 5 \text { buses }\end{array}$ \\
\hline Contingency (Line 7-8) & & 8 & 4 & $2,6,8,9$ & Bus 8 became unobserved, and solution has to change \\
\hline Shut down Bus 2 & & & 5 & $4,5,8,11,13$ & The solution changed to 5 buses \\
\hline
\end{tabular}

Table 3. Effects of differences in placement cost per bus on OPP solutions for ieee-14 bus system

\begin{tabular}{lccl}
\hline Cost Cases & Number of PMUs & Optimal Solution & Comment \\
\hline Case: Uniform Placement cost & 4 & $2,7,10,13$ & A set of 4 buses optimal solution \\
Case: Bus 2 = 2P.U. cost & 5 & $4,5,8,11,13$ & A new set of 4 buses optimal solution \\
Case: Bus $7=$ 2P.U. cost & 4 & $2,6,8,9$ & A new set of 4 buses optimal solution \\
Case: Bus 10 = 2P.U. cost & 5 & $2,7,9,11,13$ & A new set of 5 buses optimal solution \\
Case: Bus 13 = 2P.U. cost & 5 & $2,7,11,12,14$ & A new set of 5 buses optimal solution \\
Case: Bus 2, 13 = 2P.U. cost each & 6 & $3,5,7,11,12,14$ & A new set of 6 buses optimal solution \\
Case: Bus 2, 7, 10, 13 = 2P.U. cost each & 7 & $3,5,8,9,11,12,14$ & A new set of 7 buses optimal solution \\
\hline
\end{tabular}


Table 4. Combined effects of both placement contingency locations and placement cost per bus on OPP solutions for ieee-14 bus system

\begin{tabular}{lcccl}
\hline Grid State & $\begin{array}{c}\text { Optimal } \\
\text { Solution }\end{array}$ & $\begin{array}{c}\text { Number of } \\
\text { PMUs }\end{array}$ & Optimal Solution & Comment \\
\hline Normal & $2,7,10,13$ & 4 & & A set of 4 buses optimal solution \\
Contingency (Line 1-2); Bus 4 = 2P.U. cost & 5 & $1,2,7,10,13$ & A new set of 5 buses optimal solution \\
Contingency (Line 1-2); Buses 4,13 = 2p.U. cost & 6 & $1,3,7,11,12,14$ & A new set of 6 buses optimal solution \\
Contingency (Line 1-2); Bus 13 = 2P.U. cost & 6 & $4,5,7,10,12,14$ & A new set of 6 buses optimal solution \\
Contingency (Line 2-3); Bus 4 = 2P.U. cost & 5 & $1,3,7,11,13$ & A new set of 5 buses optimal solution \\
Contingency (Line 2-3); Bus 11 = 2P.U. cost & 6 & $4,5,6,7,10,13$ & A new set of 6 buses optimal solution \\
Contingency (Line 6-13); Bus 2 = 2P.U. cost & 5 & $4,5,8,11,13$ & A new set of 5 buses optimal solution \\
Contingency (Line 6-13); Buses 2,11 = 2P.U. cost & 6 & $3,5,6,7,10,13$ & A new set of 6 buses optimal solution \\
\hline
\end{tabular}

Table 5. Effects of contingency locations on OPP solutions for ieee-30 bus system

\begin{tabular}{|c|c|c|c|c|c|}
\hline Grid State & $\begin{array}{l}\text { Placement } \\
\text { Solution }\end{array}$ & $\begin{array}{l}\text { Unobserved } \\
\text { Bus }\end{array}$ & $\begin{array}{l}\text { Number of } \\
\text { PMUs }\end{array}$ & Solution & Comment \\
\hline Normal & $\begin{array}{c}1,2,6,9,10,12 \\
15,19,25,27\end{array}$ & None & 10 & & All buses were observed \\
\hline Contingency (Line 27-29) & & 30 & 10 & $\begin{array}{c}1,2,6,9,10,12,15 \\
19,25,30\end{array}$ & $\begin{array}{l}\text { Bus } 30 \text { became unobserved, and solution } \\
\text { set has to change }\end{array}$ \\
\hline Contingency (Line 19-20) & & None & 10 & $\begin{array}{c}1,2,6,9,10,12,18 \\
24,25,27\end{array}$ & A new set of 10 buses optimal solution \\
\hline Contingency (Line 2-5) & & 5 & 10 & $\begin{array}{c}3,6,7,9,10,12,15 \\
20,25,27\end{array}$ & $\begin{array}{l}\text { Bus } 5 \text { became unobserved, and solution } \\
\text { set has to change }\end{array}$ \\
\hline Contingency (Line 12-14) & & None & 10 & $\begin{array}{c}1,2,6,9,10,12,15 \\
19,25,27\end{array}$ & A new set of 10 buses optimal solution \\
\hline Contingency (Line 29-30) & & None & 10 & $\begin{array}{c}1,2,6,9,10,12,15 \\
19,25,27\end{array}$ & A new set of 10 buses optimal solution \\
\hline Contingency (Line 27-28) & & None & 10 & $\begin{array}{c}2,3,6,9,10,12,15 \\
19,25,27\end{array}$ & A new set of 10 buses optimal solution \\
\hline Shut down Bus 6 & & 7,8 & 10 & $\begin{array}{l}3,5,9,10,12,15 \\
19,25,27,28\end{array}$ & $\begin{array}{l}\text { Buses } 7 \text { and } 8 \text { became unobserved, and } \\
\text { solution set has to change }\end{array}$ \\
\hline
\end{tabular}

Table 6. Effects of differences in placement cost per bus on OPP solutions for iee-30 bus system

\begin{tabular}{|c|c|c|c|}
\hline Cost Cases & Number of PMUs & Optimal Solution & Comment \\
\hline Case: Uniform Placement cost & 10 & $1,2,6,9,10,12,15,19,25,27$ & A set of 10 buses optimal solution \\
\hline Case: Bus $6=2 P \cdot U \cdot \operatorname{cost}$ & 10 & $3,5,8,9,11,14,18,24,26,30$ & A new set of 10 buses optimal solution \\
\hline Case: Bus $27=2 P . U$. cost & 10 & $1,2,6,9,10,12,15,19,25,30$ & A new set of 10 buses optimal solution \\
\hline Case: Bus $12=2 P . U$. cost & 11 & $2,3,6,9,10,13,15,17,19,25,27$ & A new set of 11 buses optimal solution \\
\hline Case: Bus $10=2 P . U$. cost & 10 & $1,2,6,9,10,12,15,19,25,27$ & A new set of 10 buses optimal solution \\
\hline Case: Bus $10=3 P . U$. cost & 11 & $2,3,6,9,12,15,17,20,22,25,27$ & A new set of 11 buses optimal solution \\
\hline Case: Bus $2=1.5 P . U$. cost & 10 & $3,5,6,9,10,12,15,19,25,27$ & A new set of 10 buses optimal solution \\
\hline
\end{tabular}

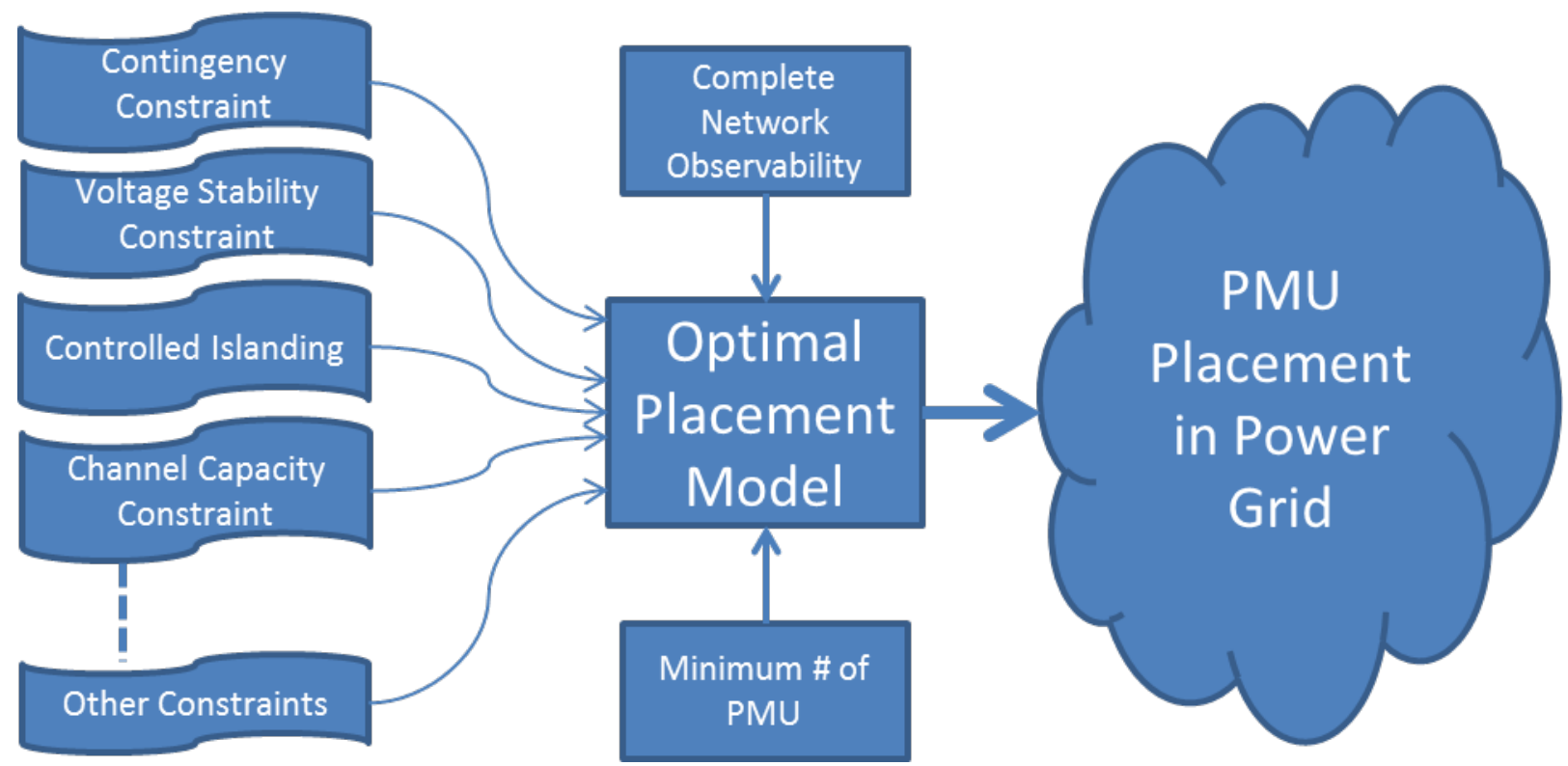

Figure 6. Schematic of an adequate OPP model 
Table 7. Optimal results achieved with some OPP models on the IEEE Test Cases

\begin{tabular}{lcccc}
\hline & IEEE 14-Bus & IEEE 30-Bus & Locations of PMUs \\
\hline Considerations & No. of PMUs & Locations of PMUs & No. of PMUs & $1,2,6,9,10,12,15,19,25,27$ \\
Normal condition & 4 & $2,7,10,13$ & 10 & $1,3,5,10,11,13,14,15,16,19,23,26,30$ \\
Considering one line outage & 7 & $1,3,6,8,9,11,13$ & 14 & $1,2,3,7,10,12,13,15,17,18,19,24,27,30$ \\
Considering one PMU outage & 7 & $1,2,4,6,9,11,13$ & 11 & $1,6,7,9,10,12,16,19,24,25,27$ \\
Considering controlled islanding & 5 & $4,7,9,11,13$ & 7 & $1,2,10,12,18,24,29$ \\
Considering ZIB & 3 & $2,6,9$ & 10 & $2,4,6,9,10,12,15,18,25,27$ \\
Considering channel limits & 5 & $2,4,6,7,9$ & 15 & $1,3,5,7,10,12,13,15,16,19,20,24,25,27,29$ \\
Considering loss of measurement & 7 & $2,4,5,6,9,10,13$ & & 13, \\
\hline
\end{tabular}

\section{Discussion}

It can be observed, from the tables, that an optimal solution achieved at the normal operating condition sometimes ceases to be the optimal solution or ceases to fulfill the constraints when certain contingency situations occur. Also, the estimated costs of PMU placement per bus in the system play a vital role in determining the number and optimal locations of PMUs to serve the synchrophasor needs of the power grid. The optimal solution is determined by the placement factors considered. Recall that OPP study is a study that is carried out once on a power grid for a permanent deployment of synchrophasor system. It means that an OPP formulation should consider all the relevant PMU placement factors in their order of relevance so as to yield a truly optimal placement solution. Figure 6 depicts a schematic of an adequate OPP model. An optimal placement model like as depicted in Figure 6 is able to identify and incorporate the necessary constraining factors so as to yield a truly optimal solution peculiar to any power grid.

\section{Conclusion}

The results above have shown that the optimal solutions achieved so far with some of the existing OPP formulations may not be truly optimal solutions being that the necessary factors were not considered. A good formulation should be able to consider all the relevant factors. It should also consider the degrees of relevance of these factors to the synchrophasor applications being deployed, so as to yield a truly optimal solution. It has, therefore, become necessary to seek ways of developing OPP formulations where relevant factors are adequately considered given their degrees of relevance to the synchrophasor applications being deployed.

A necessary step forward is to develop an optimal PMU placement model that incorporates all the necessary factors, which are considered in PMU placement, and in the way they occur in the given power grid.

\section{References}

[1] A. Phadke, "Synchronized phasor measurements in power systems," Computer Applications in Power, IEEE, vol. 6, pp. 10-15, 1993.
[2] A. Phadke, J. Thorp, and K. Karimi, "State estimlation with phasor measurements," Power Systems, IEEE Transactions on, vol. 1, pp. 233-238, 1986.

[3] E. Oleka, A. Khanal, A. Osareh, and G. Lebby, "Exploring the Challenging Issues with Synchrophasor Technology Deployments in Electric Power Grids," International Journal of Electrical, Computer, Energetic, Electronic and Communication Engineering Vol: 9, No: 9, pp. 957-960, 2015.

[4] N. M. Manousakis, G. N. Korres, and P. S. Georgilakis, "Taxonomy of PMU placement methodologies," Power Systems, IEEE Transactions on, vol. 27, pp. 1070-1077, 2012.

[5] F. Aminifar, M. Fotuhi-Firuzabad, A. Safdarian, A. Davoudi, and M. Shahidehpour, "Synchrophasor measurement technology in power systems: Panorama and state-of-the-art," Access, IEEE, vol. 2, pp. 1607-1628, 2014

[6] V. Madani, M. Parashar, J. Giri, S. Durbha, F. Rahmatian, D. Day, et al., "PMU placement considerations - a roadmap for optimal PMU placement," in Power Systems Conference and Exposition (PSCE), 2011 IEEE/PES, 2011, pp. 1-7.

[7] S. M. Mahaei and M. T. Hagh, "Minimizing the number of PMUs and their optimal placement in power systems," Electric Power Systems Research, vol. 83, pp. 66-72, 2012.

[8] V. B. Raju, T. TejaSwaroop, and R. K. Rao, "Optimal placement of phasor measurement units against PMU outage and Line outage Using advanced particle swarm optimization techniques," in Information and Communication Technologies (WICT), 2011 World Congress on, 2011, pp. 501-506.

[9] S. Chakrabarti and E. Kyriakides, "Optimal placement of phasor measurement units for power system observability," Power Systems, IEEE Transactions on, vol. 23, pp. 1433-1440, 2008.

[10] R. Sodhi, S. Srivastava, and S. Singh, "Optimal PMU placement to ensure system observability under contingencies," in Power \& Energy Society General Meeting, 2009. PES'09. IEEE, 2009, pp. 1-6.

[11] N. H. Abbasy and H. M. Ismail, "A unified approach for the optimal PMU location for power system state estimation," Power Systems, IEEE Transactions on, vol. 24, pp. 806-813, 2009.

[12] E. Makram, Z. Zhao, and A. Girgis, "An improved model in optimal PMU placement considering sensitivity analysis," in Power Systems Conference and Exposition (PSCE), 2011 IEEE/PES, 2011, pp. 1-6.

[13] L. Huang, Y. Sun, J. Xu, W. Gao, J. Zhang, and Z. Wu, "Optimal PMU placement considering controlled islanding of power system," IEEE Transactions on Power Systems, vol. 29, pp. 742-755, 2014.

[14] S. Chakrabarti, E. Kyriakides, and D. G. Eliades, "Placement of synchronized measurements for power system observability," Power Delivery, IEEE Transactions on, vol. 24, pp. 12-19, 2009.

[15] N. Theodorakatos, N. Manousakis, and G. Korres, "Optimal PMU Placement Using Nonlinear Programming," arXiv preprint arXiv:1507.05258, 2015.

[16] B. Xu and A. Abur, "Observability analysis and measurement placement for systems with PMUs," in Proc. 2004 IEEE Power System Conf, 2004, pp. 943-946.

[17] S. Jitlikhit, "Application of Phasor Measurement Units and Wide Area Monitoring System of EGAT's Network and Trend for Smart Grid," EGAT, 2010.

[18] USDOE, "Factors affecting PMU installation cost," Smart Grid Investment Grant Program, October 2014. 